\title{
Cardiopulmonary Effects of Trendelenburg Positioning in Robotic Assisted Radical Cystectomy
}

\author{
Brian Winters $^{1 *}$, Prashoban Bremjit ${ }^{2}$, Sarah Holt ${ }^{1}$, Jonathan \\ Harper ${ }^{1}$ and Jonathan Wright ${ }^{1,3}$ \\ ${ }^{1}$ Department of Urology, University of Washington School of Medicine, USA \\ ${ }^{2}$ School of Medicine, University of Washington, USA \\ ${ }^{3}$ Division of Public Health Sciences, Fred Hutchinson Cancer Research Center, USA
}

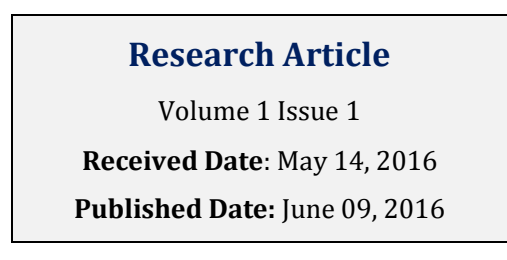

*Corresponding author: Brian Winters, Department of Urology, University of Washington School of Medicine, Seattle, WA, USA, Email: wintersb@uw.edu

\begin{abstract}
Introduction: Robotic-assisted radical cystectomy (RARC) is increasingly utilized in the management of bladder cancer (BCa) and prior studies have demonstrated steep Trendelenburg positioning can result in significant cardiopulmonary perturbations. We sought to determine the physiologic changes encountered in $\mathrm{BCa}$ patients undergoing RARC.
\end{abstract}

Materials and Methods: Consecutive patients undergoing RARC were identified with key intra-operative parameters recorded (end-tidal carbon dioxide tension (EtCO2), peak inspiratory pressure (PIP), and mean arterial pressure (MAP)) and compared between time points throughout the surgery. Multivariate linear regression was used to determine clinical and tumor characteristics associated with significant cardiopulmonary change.

Results: 61 patients were included in the analysis. Mean change inEtCO2 $(+5.2 \mathrm{mmHg})$, PIP $(+11.2 \mathrm{mmHg})$, and MAP (+14.2 $\mathrm{mmHg}$ ) were significantly elevated in Trendelenburg vs. supine positioning, respectively (all $\mathrm{p}<0.01$ ). Female gender was associated with increased PIP from T1 to T4 ( $\mathrm{p}=0.002)$ while heavy smoking history resulted in decreased PIP ( $p=0.02)$. Age range of 60-69 was associated with significantly lower EtCO2 $(p=0.02)$. There were no significant trends in MAP seen on univariate analysis. On multivariate analysis female gender remained an independent risk for elevated PIP ( $p=0.048)$ and increasing age was significantly associated with elevated MAP $(p=0.02)$. One patient required open conversion for poor pulmonary tolerance.

Conclusions: Trendelenburg positioning during RARC was associated with significant changes in several perioperative parameters however; all variables remained within clinically allowable ranges. Conversion to open cystectomy in this cohort was rare suggesting the safety of steep Trendelenburg positioning in this highly comorbid population.

Keywords: Bladder Cancer; Radical Cystectomy; Robotic; Trendelenburg 


\section{Open Access Journal of Urology \& Nephrology}

\section{Introduction}

Robotic-assisted radical cystectomy (RARC) has been increasingly utilized in the management of muscle invasive bladder cancer $[1,2]$. Compared to conventional open cystectomy, RARC offers the potential to reduce perioperative complications while maintaining comparable surgical and oncological outcomes $[3,4]$. Optimal surgical exposure in RARC is aided by placing the patient in the steep Trendelenburg position, employing a head-down tilt up to $45^{\circ}$. In combination with several hours of intra-abdominal carbon dioxide $\left(\mathrm{CO}_{2}\right)$ insufflations, this position can cause significant perturbations in respiratory and hemodynamic parameters.

$\mathrm{CO}_{2}$ pneumoperitoneum by itself has well-described effects on cardiovascular parameters including elevations in mean arterial blood pressure (MAP), heart rate (HR), central venous pressure, and pulmonary capillary wedge pressure [5-8]. Further, pneumoperitoneum combined with steep Trendelenburg positioning(as seen in the robotic-assisted laparoscopic prostatectomy (RALP) and robotic hysterectomy literature)has demonstrated altered respiratory physiology [9-11] with reductions in lung volumes and compliance $[6,9,10,12]$. Compared to patients undergoing RALPor robotic hysterectomy however, patients with bladder cancer often present with advanced age and increased comorbidities, leading to decreased cardiopulmonary functional status [13,14]. Therefore, bladder cancer patients may lack the necessary cardiopulmonary reserve to withstand prolonged pneumoperitoneum and Trendelenburg positioning.

The present study investigates changes in pulmonary and hemodynamic parameters during RARC in the steep Trendelenburg position. We hypothesize that the cardiopulmonary stress brought on by steep Trendelenburg positioning during RARC will lead to significant changes in key measures of cardiopulmonary function.

\section{Methods}

\section{Study Population}

Institutional Review Board approval was obtained (\#7892) and retrospective chart review was performed on all patients undergoing $R A R C$ for muscle invasive bladder cancer between January 2010 to December 2013 $(\mathrm{N}=64)$. Three patients required open conversion for surgical reasons and were excluded from analysis (failure to progress). One patient was converted to open due to poor pulmonary tolerance (after 2.5 hours steep Trendelenburg) but given the aims of the study was included in the analysis. Clinical variables on the remaining 61 patients were examined including demographics (race, age, gender, body mass index (BMI)), comorbidities (diabetes mellitus, pulmonary disease, cardiovascular disease, history of smoking), and tumor characteristics including clinical stage and use of neoadjuvant chemotherapy. Pulmonary disease was defined as documented chronic obstructive pulmonary disease (COPD), restrictive lung disease, obstructive sleep apnea requiring the use of a CPAP machine, or a history of abnormal pulmonary function testing. Cardiovascular disease included coronary artery disease, history of myocardial infarction, congestive heart failure, or a documented history of systolic or diastolic dysfunction. Smoking status was defined as "never smoker", "former smoker, fewer than 20 pack-years", and "active smoker or greater than 20 pack-year history".

\section{Anesthesia}

In the operating room, routine monitors were applied to each patient including 5-lead ECG, pulse oximetry, and a non-invasive automated arterial blood pressure cuff. General anesthesia was induced with intravenous propofol, fentanyl, and muscle paralytics, with transition to inhalational anesthetics as appropriate. Patients were then intubated in standard fashion and placed on volume control ventilation.

\section{Surgery}

RARC was performed using the day Vinci Surgical System (Intuitive Surgical, Sunnyvale, CA, USA) via transperitoneal approach. The abdomen was insufflated with carbon dioxide with the patient in the supine position and transitioned to steep Trendelenburg position with 45 degrees of head-down tilt after port placement. Robotic cystectomy and pelvic lymph node dissection were performed in standard fashion [15]. Once all pathologic specimens were removed, the robot was undocked and the patient was placed into supine position for extracorporeal urinary diversion.

\section{Data Collection}

Intraoperative parameters collected from anesthetic records included heart rate (HR, beats per minute), mean arterial pressure (MAP, $\mathrm{mmHG}$ ), respiratory rate (RR, breaths per minute), end-tidal carbon dioxide tension (EtCO2, mmHG), and peak inspiratory pressures (PIP, mmHG). Patient parameters were recorded 10 minutes after induction in supine position (T1), and again at one-, two-, four-, and six-hours into procedure (termed T2, T3, $\mathrm{T} 4$, and T6, respectively). Patients remained in the steep Trendelenburg position at times $\mathrm{T} 2, \mathrm{~T} 3$, and $\mathrm{T} 4$, and were 


\section{Open Access Journal of Urology \& Nephrology}

returned to supine position by T6. Clinical staging was based on TNM classification from American Joint Committee on Cancer (AJCC, Chicago, Illinois, 7th edition). Clinically acceptable ranges of commonly monitored intraoperative parameters are shown in Table 1 [16-18].

\begin{tabular}{|c|c|}
\hline \multicolumn{2}{|c|}{$\begin{array}{c}\text { Normal Cardiopulmonary Parameters Previously } \\
\text { Described }\end{array}$} \\
\hline End-tidal $\mathrm{CO}_{2}\left(\mathrm{EtCO}_{2}\right)$ & $35-45 \mathrm{mmHg}^{16}$ \\
\hline $\begin{array}{c}\text { Peak Inspiratory } \\
\text { Pressure (PIP) }\end{array}$ & $25-40 \mathrm{mmHg}^{17}$ \\
\hline $\begin{array}{c}\text { Respiratory Rate } \\
\text { (RR) }\end{array}$ & $12-20 \mathrm{bpm}^{17}$ \\
\hline Heart Rate (HR) & $60-100 \mathrm{bpm}^{18}$ \\
\hline $\begin{array}{c}\text { Mean Arterial } \\
\text { Pressure (MAP) }\end{array}$ & $70-110 \mathrm{mmHg}^{18}$ \\
\hline
\end{tabular}

Table 1: Normal Cardiopulmonary Parameters Previously Described.

Established reference ranges for collected pulmonary and hemodynamic Parameters. ${ }^{16-18} \mathrm{mmHG}$ : millimeters mercury, BPM: breaths per minute and beats per minute for $R R$ and $H R$, respectively.

\section{Statistical Analysis}

Means with standard deviations were calculated for each parameter at all time points. In depth analysis was focused on T1 and T4 (post-induction, supine position vs. four hours into procedure, steep Trendelenburg position). Differences of patient groupings were compared by univariate linear regression. Multivariate Cox regression analysis with age as a continuous variable and smoking status as a binary variable ( $\geq 20$ pack-years or active vs. $<20$ pack-years or non-smoker) was performed adjusting for age, gender, BMI, neoadjuvant chemotherapy, known pulmonary disease, and smoking status. Due to various missing data points, calculations were based on $\mathrm{N}$ of 50 , 51, and 51 for MAP, EtCO2, and PIP calculations, respectively. All statistical analyses were conducted using STATA software, Version 12 (STATA, Inc., College Station, TX).

\section{Results}

Patient demographic data are shown in Table 2. This cohort was predominantly male $(75.0 \%)$, elderly (mean age 61 (range 37-86)) and nearly half of the cohort reported active or heavy past smoking use. Eleven patients (18\%) required ICU admission after surgery. Summarized mean changes at all time points are shown in Supplementary Figure 1. Significant mean (SD, \% change) increases were seen across all parameters from T1 to T4 (all p<0.01): HR +12.6 ( $\pm 12.2 \mathrm{bpm}, 22 \%), \mathrm{MAP}+14.2( \pm$ $15.0 \mathrm{mmHg}, 23 \%) \mathrm{RR}+3.3( \pm 3.0 \mathrm{bpm}, 36 \%)$, PIP $+11.2( \pm$ $5.3 \mathrm{mg} / \mathrm{dL}, 56 \%)$, and Et $\mathrm{CO}_{2}+5.2( \pm 5.4 \mathrm{mg} / \mathrm{dL}, 18 \%)$. One patient required conversion to open cystectomy 2.5 hours in to procedure due to poor pulmonary tolerance (persistently elevated PIP to $40 \mathrm{mmHG}$ despite recruitment maneuvers, albuterol, and decreased insufflations by surgical team). In the remaining patients, all mean values remained at the upper level of clinically acceptable limits (Table 1 ).

\begin{tabular}{|c|c|}
\hline \multicolumn{2}{|c|}{ Baseline Characteristics of RARC Patients } \\
\hline Category & Patients $(\mathrm{N}=61)(\%)$ \\
\hline $\begin{array}{c}\text { Median Age at Surgery } \\
\text { (Range) }\end{array}$ & $66(37-86)$ \\
\hline \multicolumn{2}{|c|}{ Gender } \\
\hline Male & $46(75)$ \\
\hline Female & $15(25)$ \\
\hline \multicolumn{2}{|c|}{ Age (years) } \\
\hline$<60$ & $17(28)$ \\
\hline 61-69 & $20(33)$ \\
\hline$\geq 70$ & $24(39)$ \\
\hline \multicolumn{2}{|c|}{ Body Mass Index $\left(\mathrm{kg} / \mathrm{m}^{2}\right)$} \\
\hline$<30$ & $41(67)$ \\
\hline$\geq \mathbf{3 0}$ & $20(33)$ \\
\hline \multicolumn{2}{|c|}{ Clinical Stage } \\
\hline$\leq \mathrm{T} 2$ & $41(67)$ \\
\hline$>\mathrm{T} 2$ & $20(33)$ \\
\hline \multicolumn{2}{|c|}{ Smoking Status } \\
\hline Never Smoker & $19(31)$ \\
\hline <20 Pack-Year History & $13(21)$ \\
\hline $\begin{array}{c}\geq 20 \text { Pack-Year History } \\
\text { or Active }\end{array}$ & $29(48)$ \\
\hline \multicolumn{2}{|c|}{ Comorbidities } \\
\hline Pulmonary Disease & $11(18)$ \\
\hline Diabetes mellitus & $9(15)$ \\
\hline Cardiovascular Disease & $11(18)$ \\
\hline
\end{tabular}

Table 2: Baseline Characteristics of RARC Patients. 


\section{Open Access Journal of Urology \& Nephrology}

Numbers in parentheses equal \% unless otherwise noted. RARC: robotic assisted radical cystectomy; $\mathrm{Kg} / \mathrm{m} 2$ : kilograms per meters squared.

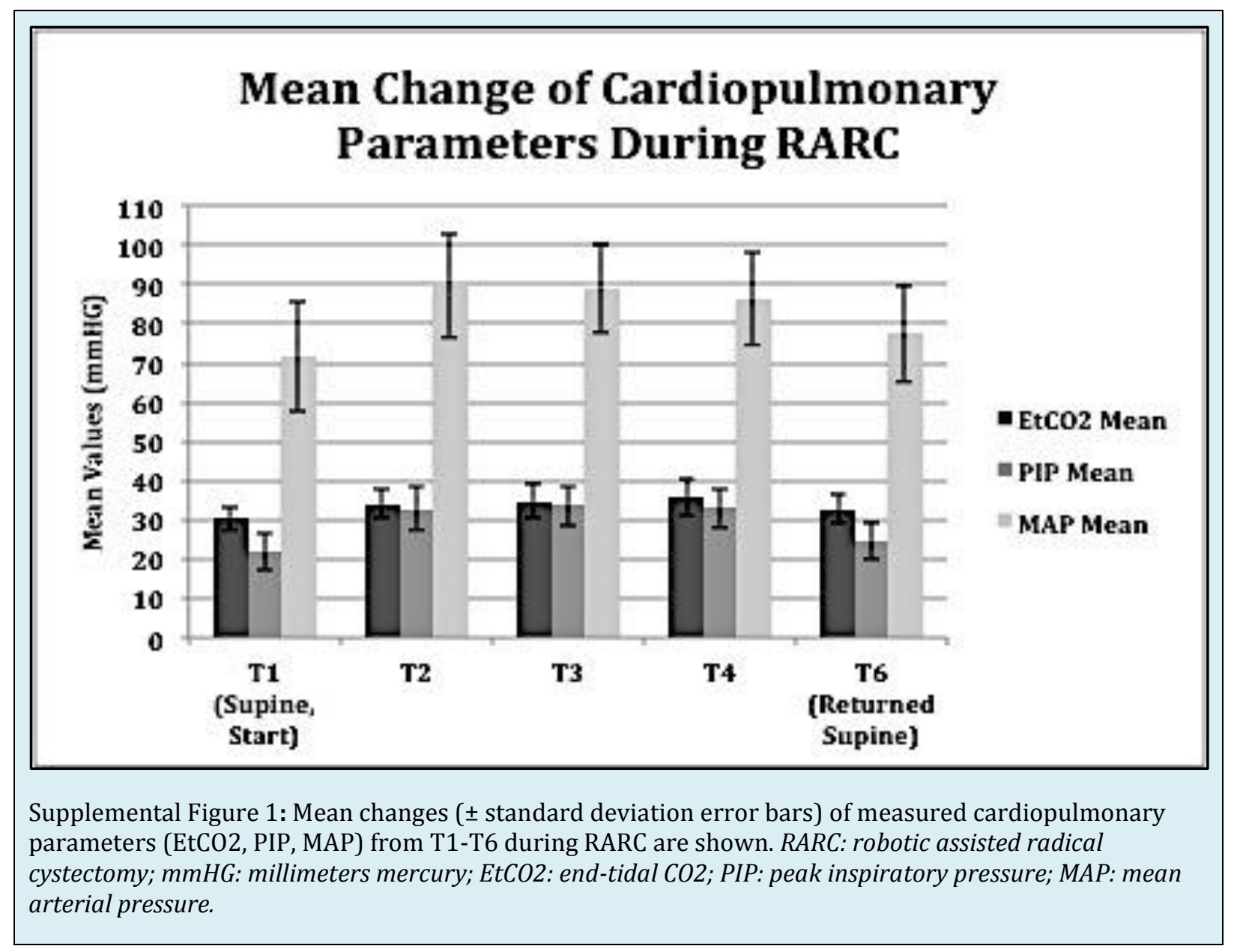

\section{Pulmonary parameters}

On univariate analysis (Table 3), female gender was associated with significant increases in PIP from T1 to T4 $(\mathrm{p}=0.002)$ while heavy smoking status was associated with significantly decreased PIP ( $\mathrm{p}=0.02)$. Patients aged 60-69 years of age at the time of surgery were noted to have a significantly decreased EtCO2 compared to the other age groupings $(\mathrm{p}=0.03)$.
On multivariate analysis, female gender remained significantly associated with an adjusted mean increase of $4.2 \mathrm{mmHG}$ in PIP compared to men $(\mathrm{p}=0.04)$. There were no significant findings relative to $\mathrm{Et}^{\mathrm{CO}_{2}}$ change on multivariate analysis. 


\section{Open Access Journal of Urology \& Nephrology}

\begin{tabular}{|c|c|c|c|}
\hline \multicolumn{4}{|c|}{ Cardiopulmonary Changes During RARC } \\
\hline & MAP Mean $\Delta$ & ETC02 Mean $\Delta$ & PIP Mean $\Delta$ \\
\hline & $\mathrm{T} 1-\mathrm{T} 4(\mathrm{~N}=50)$ & T1-T4 (N=51) & $\mathrm{T} 1-\mathrm{T} 4(\mathrm{~N}=51)$ \\
\hline \multicolumn{4}{|c|}{ Gender } \\
\hline Male & $14.5 \pm 11.5$ & $5.0 \pm 5.9$ & $10.0 \pm 5.1$ \\
\hline Female & $13.2 \pm 23.7$ & $5.8 \pm 3.6$ & $15.2 \pm 3.7^{*}$ \\
\hline \multicolumn{4}{|c|}{ Age (years) } \\
\hline$<60$ & $12.9 \pm 19.5$ & $7.8 \pm 5.4$ & $10.7 \pm 5.6$ \\
\hline $60-69$ & $8.5 \pm 15.6$ & $3.4 \pm 4.7^{*}$ & $11.7 \pm 6.1$ \\
\hline$\geq 70$ & $19.3 \pm 9.2$ & $5.0 \pm 5.5$ & $11.2 \pm 4.6$ \\
\hline \multicolumn{4}{|c|}{ Body Mass Index (kg/m2) } \\
\hline$<30$ & $12.6 \pm 13.5$ & $5.6 \pm 5.7$ & $11.1 \pm 5.6$ \\
\hline$\geq 30$ & $17.2 \pm 17.6$ & $4.6 \pm 5.0$ & $11.4 \pm 4.9$ \\
\hline \multicolumn{4}{|c|}{ Clinical Stage } \\
\hline$\leq \mathrm{T} 2$ & $11.9 \pm 14.8$ & $4.6 \pm 4.3$ & $10.9 \pm 5.5$ \\
\hline$>\mathbf{T} 2$ & $20.1 \pm 14.5$ & $6.9 \pm 7.6$ & $12.0 \pm 4.7$ \\
\hline \multicolumn{4}{|c|}{ Smoking Status } \\
\hline Never & $14.4 \pm 20.5$ & $5.1 \pm 4.0$ & $13.5 \pm 4.2$ \\
\hline$<20$ Pack Years & $16.5 \pm 13.6$ & $6.5 \pm 3.4$ & $11.9 \pm 6.8$ \\
\hline$\geq 20$ Pack Years or Active & $13.0 \pm 11.9$ & $4.7 \pm 6.7$ & $9.6 \pm 4.7^{*}$ \\
\hline \multicolumn{4}{|c|}{ Pulmonary Disease } \\
\hline No & $14.3 \pm 15.9$ & $4.9 \pm 3.8$ & $11.7 \pm 5.1$ \\
\hline Yes & $13.8 \pm 11.1$ & $6.8 \pm 10.3$ & $9.0 \pm 6.0$ \\
\hline \multicolumn{4}{|c|}{ Cardiovascular Disease } \\
\hline No & $14.3 \pm 15.7$ & $5.4 \pm 5.7$ & $11.6 \pm 5.3$ \\
\hline Yes & $13.7 \pm 4.1$ & $4.2 \pm 1.4$ & $9.7 \pm 1.8$ \\
\hline \multicolumn{4}{|c|}{ Neoadjuvant Chemotherapy } \\
\hline No & $11.7 \pm 12.8$ & $4.0 \pm 4.5$ & $10 \pm 5.7$ \\
\hline Yes & $15.3 \pm 16.0$ & $5.7 \pm 5.7$ & $11.8 \pm 5.1$ \\
\hline
\end{tabular}

Table 3: Cardiopulmonary Changes during RARC.

Unadjusted mean change in MAP, EtCO2, and PIP from T1 (supine, post-induction) to T4 (steep Trendelenburg position, 4 hours in to cystectomy). Data expressed as means \pm standard deviation. Due to missing data, the number of patients for calculation is clarified in each column (N). P-values calculated with linear regression, with top row as referent within each covariate. ${ }^{*}$ Denotes significance $(\mathrm{p}<0.05)$. RARC: robotic assisted radical cystectomy; $\mathrm{Kg} / \mathrm{m}^{2}$ : kilograms per meters squared. 


\section{Open Access Journal of Urology \& Nephrology}

\section{Hemodynamic parameters}

Univariate analysis of MAP change revealed no statistically significant findings though increases relative to BMI, clinical stage, and neoadjuvant chemotherapy were seen. Interestingly, there was no linear trend based on age groupings on univariate analysis however, on multivariate analysis, with age as a continuous variable, there was a significant increase in MAP with increasing age $(\mathrm{p}=0.02)$.

\section{Discussion}

RARC offers a number of potential advantages to traditional open radical cystectomy, including reduction of perioperative bleeding and pain while maintaining oncological results and efficacy $[3,4,19,20]$. As a result, RARC has been increasingly utilized in the management of muscle invasive bladder cancer [1,2]. In order to provide optimal surgical exposure, this procedure requires positioning in steep Trendelenburg for several hours with known alterations in cardiovascular physiology $[5,10,12]$. The safety of steep Trendelenburg positioning in bladder cancer patients undergoing RARC, who often present with chronic cardiovascular and pulmonary comorbidities $[13,14]$ has not been well described. The present study demonstrated prolonged positioning in steep Trendelenburg during RARC is associated with significant elevations in key pulmonary and hemodynamic parameters (PIP, EtCO2, and MAP) however these changes remain in clinically acceptable ranges. These data support that RARC is indeed safe in this population of high comorbidity.

Significant elevations in PIP following prolonged steep Trendelenburg position is consistent with findings in other studies $[6,10,12,21]$ and may be dependent upon the type of ventilation used (volume vs. pressure control) [22]. Subjecting the patient to steep head-down tilt causes compression of the thoracic cavity by exacerbating the pneumoperitoneum-induced cephalad displacement of the diaphragm and abdominal contents. These factors reduce the compliance of the lung-chest wall system, leading to increased airway resistance and resultant elevated inspiratory pressures. In the present study, female gender was found to be a significant risk factor for change in PIP. Women characteristically have lower lung volumes and capacities than men even when matched for height and weight [23]. Further, lung volumes and functional residual capacity (FRC) are reduced intraoperatively secondary to anesthesia, paralysis, pneumoperitoneum, and head-down tilt [9] and it is possible that the lower baseline FRC seen in women is more susceptible to falling below the closing capacity of the lungs, resulting in higher PIPs. The finding of decreased PIP on univariate analysis in heavy smokers compared to non-smokers may be explained by altered pulmonary physiology brought on by cigarette smoking. Tobacco abuse leads to loss of elastic tissue and lung recoil (increased compliance), which may lead to comparatively lower PIP levels.

The significant increase in Et $\mathrm{CO} 2$ from $\mathrm{T} 1$ to $\mathrm{T} 4$ observed in the presentseries is consistent with findings from other studies $[10,12,24]$. Kalmar et al. studied the correlation between Et $\mathrm{CO} 2$ and $\mathrm{PaCO} 2$ in patients subjected to steep Trendelenburg positioning, and found an actual underestimation of true arterial carbon dioxide tension with increasing levels of Et CO2 [12]. Therefore, management of ventilator settings to maintain EtCO2 within safe limits is especially critical in these patients. In our study, higher peak EtCO2 levels were found in patients with known pulmonary disease, likely a result of these patients exhibiting carbon dioxide retention at baseline. Regardless, mean EtCO2 remained within safe limits at all times in our series.

MAP was notably increased ( $+14.2 \mathrm{mmHG})$ from $\mathrm{T} 1$ to T4 with steep Trendelenburg positioning. This increase may be caused by both pneumoperitoneum (increase in intra-abdominal pressure increases after load through compression of the aorta [25]) and Trendelenburg positioning itself (increased sympathetic tone is seen following pneumoperitoneum and Trendelenburg as a response to hypercapnia and peritoneal stretching [26]). Additionally, there is evidence other mediators including humoral effectors may play a role in this physiology [26]. These findings are consistent with previously reported studies involving RALP $[6,10,12]$.The present study found older age to be associated with elevations in MAP on multivariate analysis. Elderly patients may lack the physiologic reserve necessary to tolerate large perturbations in blood pressure due to the attenuation of vascular responsiveness and the baroreceptor reflex seen with aging.

There are several limitations to this study. Due to the retrospective nature, heterogeneous anesthesia protocols were used between different patients and the anesthetic agents used, dosages, and ventilator settings throughout each case were chosen as deemed necessary to optimize patient health and safety. In addition, only routinely collected parameters were available to us, leaving valuable advanced markers such as pulmonary dynamics, cardiac output, systemic vascular resistance, and arterial blood gas tensions unavailable. In addition, documentation of insufflations relative to patient positioning was often not charted making us unable to distinguish the independent effects of pneumoperitoneum and steep Trendelenburg positioning. Finally, this is a 


\section{Open Access Journal of Urology \& Nephrology}

single institutional review where inherent selection bias cannot be ruled out. Our cohort experienced only one patient requiring open conversion due to pulmonary difficulties, which may be an underestimation of the true population risk.

In conclusion, this retrospective study of patients undergoing RARC revealed prolonged pneumoperitoneum and steep Trendelenburg positioning significantly altered intraoperative cardiopulmonary dynamics yet all parameters remained at the upper level of clinically acceptable limits. This supports the safety of RARC in this highly comorbid population of bladder cancer patients. Future prospective trials will help further quantify the physiological effects of steep Trendelenburg positioning in patients undergoing RARC, as well as evaluate pertinent risk factors for adverse perioperative outcomes.

\section{Acknowledgement}

Received financial support from the Howard J Cohen Bladder Cancer Foundation.

\section{References}

1. Siren EK, Haapasalo MP, Waltimo TM, Orstavik D (2004) In vitro antibacterial effect of calcium hydroxide combined with chlorhexidine or iodine potassium iodide on Enterococcus faecalis. Eur J Oral Sci 112(4): 326-331.

2. Almyroudi A, Mackenzie D, McHugh S, Saunders WP (2002) The effectiveness of various disinfectants used as endodontic intra canal medicaments: an in vitro study. J Endod 28(3): 163-167.

3. Fava LR, Saunders WP (1999) Calcium hydroxide pastes: classification and clinical indications. Int Endod J 32(4): 257-282.

4. Lambrianidis T, Kosti E, Boutsioukis C, Mazinis M (2006) Removal efficacy of various calcium hydroxide/chlorhexidine medicaments from the root canal. Int Endod J 39(1): 55-61.

5. Menezes MM, Valera MC, Jorge AO, Koga-Ito CY, Camargo $\mathrm{CH}$, et al. (2004) In vitro evaluation of the effectiveness of irrigants and intracanal medicaments on microorganisms. Int Endod J 37(5): 311-319.

6. Ebert J, Roggendorf MJ, Frank K, Petschelt A (2008) Antimicrobial activity of various 'active' gutta percha points against Enterococcus faecalis in simulated root canals. Int Endo J 41(3): 249-257.

7. Vijay R, Makam S, Shashikala K (2010) Evaluation of antimicrobial efficacy of chlorhexidine gutta percha and calcium hydroxide gutta percha against Enterococcus faecalis - an in vitro study. Streamdent 1(3): 209- 213.

8. Vianna ME, Gomes BP, Sena NT, Zaia AA, Ferraz CC, et al. (2005) In vitro evaluation of the susceptibility of endodontic pathogens to calcium hydroxide combined with different vehicles. Braz Dent J 16(3): 175-180.

9. Gomes BP, Souza SF, Ferraz CC, Teixeira FB, Zaia AA, et al. (2003) Effectiveness of 2\% chlorhexidine gel and calcium hydroxide against Enterococcus faecalis in bovine root dentine in vitro. Int Endod J 36(4): 267275.

10. Marshell FJ, Savoic FL (1967) Efficiency of endodontic culturing procedures using wet and dry paper points. Oral Surg Oral Med Oral Pathol 23(6): 806-810.

11. Mackie, McCartney (1996) Practical Medical Microbiology. (14 $4^{\text {th }}$ edn), Churchill living stone, Edinburgh, UK, pp. 107-108.

12. Monica Cheesbrough (1984) Medical laboratory manual for topical countries. Volume II, Cambridge University Press, UK, pp. 53,175-178.

13. Monica, Cheesbrough (1999) District laboratory practice in tropical countries. Volume II, Cambridge University Press, UK, pp. 60, 83,126,128.

14. Sundqvist G (1992) Ecology of the root canal flora. J Endod 18(9): 427-430.

15. Baumgartner JC, Falker WA (1991) Bacteria in the apical 5-mm of infected root canals. J Endod 17(8): 380-383.

16. Dalton BC, Orstavik D, Phillips C, Pettiette M, Trope M (1998) Bacterial reduction with nickel titanium rotary instrumentation. J Endod 24(11): 763-767.

17. Ebert J, Roggendorf MJ, Frank K, Petschelt A (2008) Antimicrobial activity of various 'active'gutta-percha points against Enterococcus faecalis in simulated root canals. Int Endod J 41(3): 249-257.

18. Podbielski A, Boeckh C, Haller B (2000) Growth 


\section{Open Access Journal of Urology \& Nephrology}

inhibitory activity of gutta-percha points containing root canal medications on common endodontic bacterial pathogens as determined by an optimized quantitative in vitro assay. J Endod 26(7): 398-403.

19. Singh H, Kapoor P (2014) A Comparative evaluation of Antibacterial Efficacy of 'Activ Points'And 'Combi Points'as Intra-Canal Medicaments against Enterococcus faecalis: An Ex Vivo Study. Oral Health Dent Manag 13(3): 610-613.

20. Estrela C, Pimenta FC, Ito IY, Bammann LL (1999) Antimicrobial evaluation of calcium hydroxide in infected dentinal tubules. J Endod 25(6): 416-418.

21. Safavi KE, Nichols FC (1993) Effect of calcium hydroxide on bacterial lipopolysaccharide. J Endod 19(2): 76-78.

22. Tanomaru JM, Pappen FG, Tanomaru Filho M, Spolidorio DM, Ito IY (2007) In vitro antimicrobial activity of different gutta-percha points and calcium hydroxide pastes. Braz Oral Res 21(1): 35-39.

23. B Naik, S Shetty, M Yel (2013) Antimicrobial activity of gutta-percha points containing root canal medications against E. faecalis and Candida albicans in simulated root canals- An in vitro study. Endodontology 25(2): 8-18.

24. Alagarsamy A, Ebenezar AVR, Srinivasan MR, Mohan AG , Kumar S (2013) Effectiveness of calcium hydroxide plus points and chlorhexidine activ points against Enterococcus faecalis by agar diffusion test: An in vitro study. JRD 1(1): 18-21.

25. Holland R, Murata SS, Dezan E, Garlipp O (1996) Apical leakage after root canal filling with an experimental calcium hydroxide gutta-percha point. J Endod 22(2): 71-73.

26. Larsen MJ, Horsted-Bindslev P (2000) A laboratory study evaluating the release of hydroxyl ions from various calcium hydroxide products in narrow root canal like tubes. Int Endod J 33(3): 238-242.

27. Economides N, Koulaouzidou EA, Beltes P, Kortsaris $\mathrm{AH}$ (1999) In vitro release of hydroxyl ions from calcium hydroxide gutta percha points. J Endod 25(7): 481-482.

28. Ardeshna SM, Qualtrough AJ, Worthington HV (2002) An in vitro comparison of $\mathrm{pH}$ changes in root dentine following canal dressing with calcium hydroxide points and a conventional calcium hydroxide paste. Int Endod J 35(3): 239-244.

29. Azabal-Arroyo M, Menasalvas-Ruiz G, Martin-Alonso J, Arroquia JJ, Vega-del Barrio JM (2002) Loss of hydroxyl ions from gutta percha points with calcium hydroxide in their composition: an in vivo study. J Endod 28(10): 697-698.

30. Schafer E, Albehiassi A (2000) pH changes in root dentin after root canal dressing with gutta percha points containing calcium hydroxide. J Endod 26(11): 665-667.

31. Fuss Z, Rafaeloff R, Tagger M, Szajkis S (1996) Intracanal $\mathrm{pH}$ changes of calcium hydroxide pastes exposed to carbon dioxide in vitro. J Endod 22(7): 362-364.

32. Al-Nazhan S (2002) Antimicrobial activity of extracts of calcium hydroxide points. Oral Surg Oral Med Oral Pathol Oral Radiol Endod 93(5): 593-595.

33. Greenstein G, Berman C, Jaffin R (1986) Chlorhexidine an adjunct to periodontal therapy. J. Periodontal 57(6): 370-377.

34. Heling I, Sommer M, Steinberg D, Friedman M, Sela MN (1992) Microbiological evaluation of the efficacy of Chlorhexidine in a sustained-release device for dentin sterilization. Int Endod J 25(1): 15-19.

35. Lenet BJ, Komorowski R, Wu XY, Huang J, Grad H, et al. (2000) Antimicrobial substantivity of Bovine root dentin exposed to different chlorhexidine delivery vehicles. J Endod 26(11): 652-655.

36. Barthel CR, Zimmer S, Zilliges S, Schiller R, Gobel UB, et al. (2002) In situ antimicrobial effectiveness of chlorhexidine and calcium hydroxide: gel and paste versus gutta percha points. J Endod 28(6): 427-430. 